Sie beeinflusst Krankheit und Gesundheit des Kindes für sein gesamtes späteres Leben und kann darüber hinaus an die nächste Generation vererbt werden. Frühe Lebensstilberatung und eine optimale Nährstoffsupplementation während der Schwangerschaft sind demnach essenziell für eine zeitgemäße Vorsorge.

? Um welche Nährstoffsupplementation geht es?

Leißling: Fest steht: Ein Teller Gemüsesuppe bringt es nicht. Vielmehr ist die Versorgung mit Mikronährstoffen wie Folsäure, Selen, Vitamin E, Vitamin D, Coenzym Q10 oder Omega-3-Fettsäuren entscheidend - ebenso wie Dosierung und Zeitpunkt der Substitution. Der Aufklärungsbedarf ist groß: So zeigte eine aktuelle Studie der Technischen Universität München, dass nur ein Drittel der befragten Frauen Folsäure mindestens vier Wochen vor Schwangerschaftseintritt zugeführt hatte und damit zu spät, um das Risiko für Neuralrohrdefekte zu senken.

? Wird nicht vor der Einnahme von Vitamin D auch gewarnt?

Leißling: Selbstverständlich sollten vor jeder Verordnung entsprechende Laboruntersuchungen stehen, um den individuellen Bedarf zu bestimmen und die Dosierung festzulegen. Sinnvoll ist die Bestimmung von Vitalstoffen wie Zink und Selen, die Bestimmung des Homocysteins und eben des Vitamin-D-Status, um den es auch bei Schwangeren häufig schlecht steht. Eine
Studie des Instituts für Ernährungswissenschaft der Justus-Liebig-Universität Gießen mit 261 Schwangeren, die jüngst im „British Journal of Nutrition" veröffentlicht wurde, zeigte: Mehr als 95 Prozent der werdenden Mütter wiesen im Winter einen Vitamin-DSpiegel unterhalb des empfohlenen Richtwertes auf; rund die Hälfte der untersuchten Schwangeren besaß auch im Sommer zu wenig Vitamin D. Damit einher geht auch ein vierfach höheres Risiko für eine Sectio caesarea. Umgekehrt kann ein guter mütterlicher Vitamin-D-Status das Risiko für eine Präeklampsie um 30 Prozent reduzieren und beim Kind das Auftreten von Autoimmunerkrankungen wie Diabetes mellitus Typ 1, multiple Sklerose oder rheumatoide Arthritis, von psychischen Störungen und von Atemwegserkrankungen wie Asthma bronchiale oder allergische Rhinitis senken.

? Wie beurteilen Sie Fortschritte in der Vorsorge durch das Screening auf Gestationsdiabetes mellitus?

Leißling: Sicher stellt das 2011 endlich eingeführte Screening eine teilweise Verbesserung dar. Die Verwendung des 50-g-Suchtests statt des von den Fachgesellschaften empfohlenen 75-g-oGTT ist allerdings nur bedingt als Fortschritt zu bewerten, denn damit wird etwa ein Viertel der Fälle nicht erkannt. Die Diagnose eines Schwangerschaftsdiabetes ist aber ungemein wichtig, da nach einem Gestationsdiabetes etwa 35 Prozent der Frauen innerhalb von zehn
Jahren einen manifesten Diabetes entwickeln. Zudem steigen aufgrund der epigenetischen Effekte nach intrauteriner Exposition gegenüber erhöhten Glukosekonzentrationen die Risiken der Kinder, bereits in jungen Jahren an Übergewicht bis hin zur Adipositas, Diabetes, metabolischem Syndrom und erhöhtem Blutdruck zu erkranken.

? Welche Empfehlung geben Sie Ihren Kollegen mit Blick auf eine zeitgemäße Schwangerenvorsorge?

Leißling: Wir brauchen eine andere Schwangerenvorsorge als vor 20 Jahren, und da sehe ich uns Gynäkologen in der Pflicht. Wir sind zum Wohle von Mutter und Kind in der Lage, eine nachweislich bessere Vorsorge zu leisten und das sollten wir auch tun. Voraussetzung dafür ist natürlich unsere ärztliche Qualifikation durch entsprechende Fortbildungen. Mit modernen Methoden, die wir wirtschaftlich erfolgreich anwenden können, schaffen wir eine Winwin-Situation für Arzt und Patientin. Dazu zählt beispielsweise auch die Berücksichtigung der Mund- und Darmflora mit ihrer Bedeutung für die Fruchtbarkeit und das Immunsystem. Entsprechende Workshops stehen für den Herbst 2016 auf dem Fortbildungsprogramm der GenoGyn. Weitere Informationen erhalten Interessierte in unserer Geschäftsstelle (Telefonnummer 0221 / 940505 390).

! Das Interview führte die Pressestelle der GenoGyn.

\title{
Exklusiv für Mitglieder: GenoGyn startet Serviceportal mit Online-Frauenarztsuche
}

$\mathrm{M}$ it www.frauenarzt-suche.de hat die GenoGyn ein neues Serviceportal für Patientinnen gestartet, das neben Informationen rund um die Gynäkologie, die Geburtshilfe und die Präventionsmedizin, als Herzstück eine OnlineFrauenarztsuche bietet, in der ausschließlich registrierte GenoGyn-Praxen angezeigt werden. Nach verschiedenen Suchkriterien - Name des Arztes, Tätigkeitsschwerpunkte sowie Stadtname oder Postleitzahl - können in der Arztsuche passende Praxen herausgefiltert werden. Damit trägt der Vorstand der GenoGyn den digitalen Gewohnheiten vieler Patientinnen Rechung, online einen Frauenarzt in Wohnortnähe oder eine Praxis mit speziellen Leistungsschwerpunkten zu suchen. Gleichzeitig stärkt die Ärzteorganisation die Internetpräsenz ihrer Mitglieder-Praxen, denn für die in der Datenbank registrierten Praxen ist es ein werbewirksames Präsentationsforum, das sich durch die Anbindung an Google und Facebook positiv auf die verlinkten praxiseigenen Internetseiten auswirken kann, etwa durch ein besseres Ranking. Interessier-

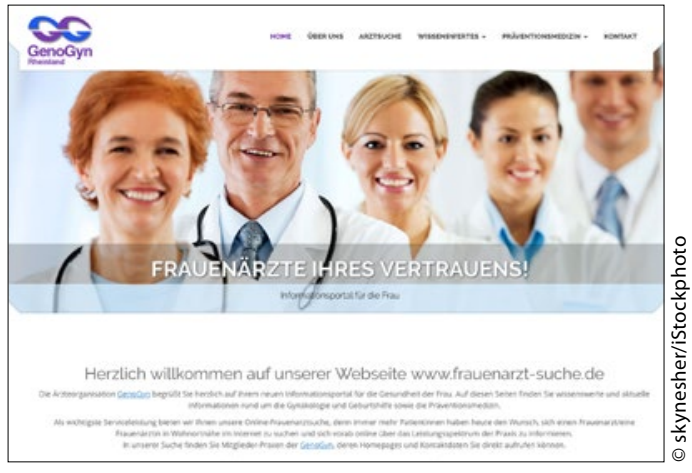

te GenoGyn-Mitglieder können die kostenlose Registrierung jederzeit auf der Homepage der GenoGyn unter www.genogyn.de vornehmen oder sich in der Geschäftstelle unter der Telefonnummer 0221/94 0505390 informieren. 